\title{
O periódico Ciência da Informação na década de 90: um retrato da área refletido em seus artigos
}

Suzana Pinheiro Machado Mueller

Professora, Programa de Pós-Graduação em Ciência da Informação da Universidade de Brasília

\section{Cláudia Maria Pinho de Abreu Pecegueiro}

Aluna do Curso de Mestrado em Ciência da Informação da Universidade de Brasília

\section{Resumo}

Os artigos publicados na revista Ciência da Informação (editada pelo IBICT) no período 1990-1999 são examinados com o objetivo de identificar certas características as quais, dada a importância desse periódico no panorama da área no Brasil, podem ser consideradas indicadores significativos da produção científica da área: volume de pesquisas, temas predominantes, identificação e número de autores segundo grupos temáticos, cooperação entre autores e produtividade individual. Foi feita ainda uma comparação entre a freqüência dos temas dos artigos examinados e os descritores registrados no LISA no mesmo período, verificando-se diferenças significativas de ênfase.

\section{Palavras-chave}

Periódicos científicos - Ciência da Informação; Artigos científicos - Ciência da Informação; Ciência da Informação temática dos artigos.

\section{The journal Ciência da Informação in the decade of 90}

\begin{abstract}
Articles published in the Brazilian periodical Ciência da Informação (issued by IBICT) between 1990-1999 are examined to identify predominant subjects and to group authors according to interest; other characteristics such as collaborative authorship and author's productivity were also identified. A tentative comparison was made between subjects of these articles and descriptors in LISA in the same period, which showed marked differences. Given the role the periodical Ciência da Informação has played in the area, results may constitute important indicators of research activity for the period in that area.
\end{abstract}

\section{Keywords}

Scientific periodicals - Brazil: Ciência da Informação; Information Science - periodical articles - subjects.

\section{INTRODUÇÃO}

Estudos sobre revistas científicas não são raros na literatura profissional. Entre esses estão dois estudos publicados em 1990 e 1993, por Javerlin \& Vakkari, que tiveram muita influência sobre estudos posteriores, estabelecendo um modelo de análise. Esses autores examinaram artigos publicados em um grupo de periódicos internacionais de elite, na área de ciência da informação, para identificar assuntos predominantes, metodologias e estratégias empregadas nas pesquisa relatadas. No Brasil, sua metodologia foi seguida, entre outros, por Teixeira (1997), que a adaptou para estudar dissertações de mestrado defendidas por alunos do Programa de PósGraduação em Ciência da Informação da Universidade de Brasília.

O presente artigo segue a linha geral proposta por Javerlin $\&$ Vakkari e faz uso de uma tabela de assunto proposta por Teixeira, adaptada. O objetivo é identificar características na literatura científica brasileira da área de ciência da informação e assim contribuir para o entendimento da área no país. Tem como objeto de estudo a revista Ciência da Informação, publicada sem interrupções desde 1972 pelo Instituto Brasileiro de Informação em Ciência e Tecnologia (Ibict). O período estudado é a década de 90 , tendo sido incluídos todos os artigos publicados nos fascículos datados de 1990 a 1999 (volumes 19 a 28 ). Esses artigos foram examinados com o objetivo de identificar:

- volume e distribuição dos artigos ao longo da década;

- características da autoria: identificação, colaboração e produtividade;

- tendência temática dos artigos;

- relação de temas dos artigos com temas internacionais indexados, no mesmo período, no Library Information Science (LISA);

- grupo de autores por interesse temático

Vários estudos sobre a produção de periódicos científicos no Brasil têm revelado problemas relacionados à qualidade de editoração, falta de recursos financeiros e apoio institucional, irregularidade (atraso) nas edições, 
problemas na distribuição e comercialização ${ }^{1}$. Mas a Ciência da Informação sempre teve suficiente apoio institucional do Ibict, o que lhe permitiu publicação ininterrupta e crescimento. Foi iniciada com periodicidade semestral que vigorou até o ano de 1991, quanto passou a quadrimestral. Segundo Mueller, Campello \& Dias (1996), “...talvez tenha sido a interrupção da Revista de Biblioteconomia de Brasília, entre 1990 a 1994, e o atraso quase crônico das demais que tenha estimulado esse aumento da periodicidade da Ciência da Informação" (p.340).

O tema proposto neste artigo é pertinente para uma área que busca conhecer-se melhor, e a fonte escolhida - o artigo publicado - certamente reflete as preocupações e interesses dessa área. A revista ciência da informação é considerada um dos periódicos de maior credibilidade na área da ciência da informação no Brasil. Com 28 anos de publicação sem interrupção, seu prestígio e influência justificam sua escolha. O período escolhido para análise, década de 90, é significativo, pois testemunhou o desenvolvimento e difusão das tecnologias de informação que provocaram modificações profundas na comunicação científica e no próprio objeto de estudo da área, tais como a aceleração na geração e disseminação da informação, modificações na forma de tratamento e armazenagem e a generalização do uso de meios eletrônicos primeiramente na comunicação informal e mais recentemente, também formal:

"A última década foi marcada por mudanças tecnológicas incessantes e dramáticas nos três principais ramos das tecnologias de informação: a informática, as comunicações e os conteúdos. Assistimos ao surgimento dos computadores pessoais, das redes mundiais de transmissão por pacotes, do disco ótico e outros meios de armazenamento em massa, da tecnologia de vídeo interativo, das técnicas de tratamento de imagens, das técnicas de digitalização com o uso dos scanners, das tecnologias de computação gráfica e ao crescimento de grandes bases de dados públicas e privadas". Chen (1999, p. 26)".

Espera-se que essa análise venha contribuir na avaliação do desenvolvimento científico alcançado pela ciência da informação no Brasil.

\section{Metodologia e Resultados}

Como unidade de análise foram tomados somente os artigos publicados na revista Ciência da Informação no período 1990-1999, desprezando-se as demais seções: Editorial, Entrevista, Ponto de Vista, Relatos de Experiência.

\footnotetext{
${ }^{1}$ Veja por exemplo: Mostafa (1996), Mueller (1999), Miranda A. (1981), Miranda D e Pereira (1996).
}

FIGURA 1

Artigos por volume e fascículo

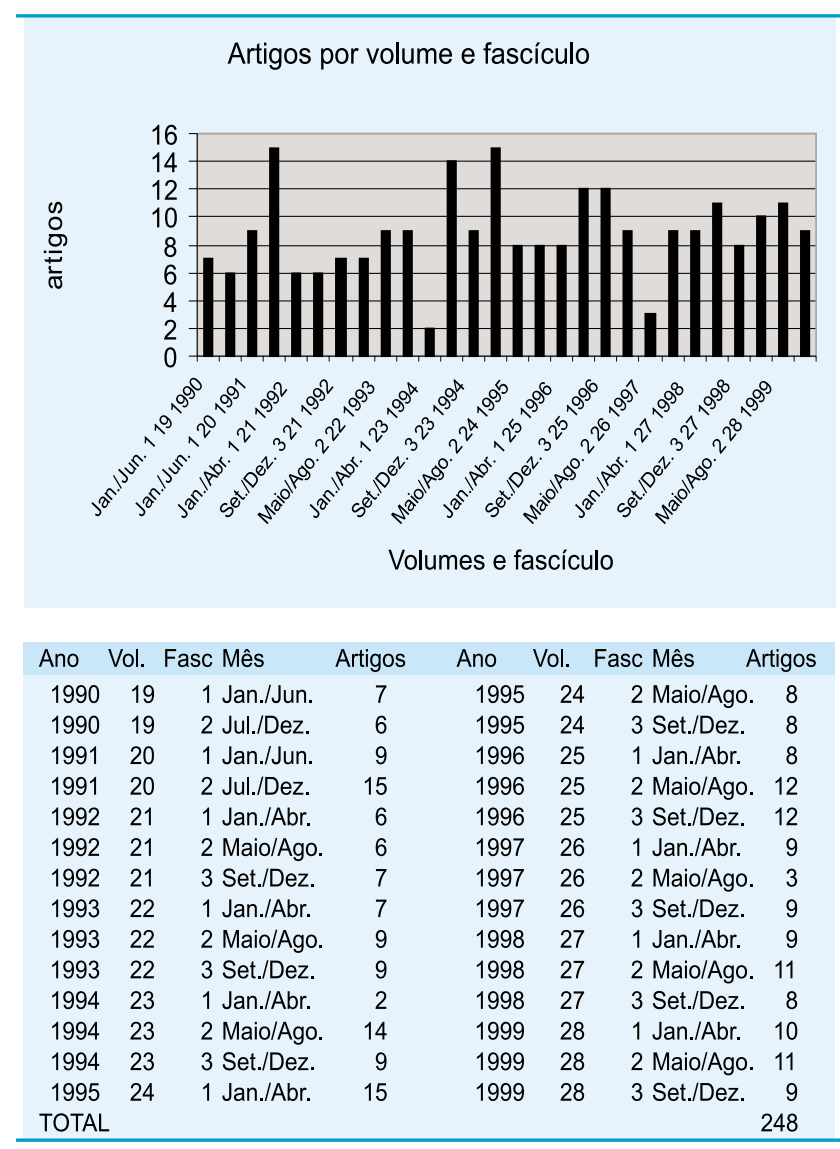

Fonte: Ciência da Informação, 1990-1999

O universo totaliza 248 artigos, assinados por 270 autores. Os artigos foram classificados por ano de publicação, volume, número e mês, conforme mostra a figura 1. Para obtenção dos dados, foram utilizadas as informações contidas na primeira página de cada artigo: título, autor(es), resumo e palavra(s)-chave.

\section{Número de artigos por fascículo}

Os dados relacionados ao volume de artigos publicados e a sua distribuição ao longo da década foram coletados com a intenção de se obter um indicador da atividade científica da área, ainda que parcial. Os 248 artigos publicados estão distribuídos em 28 fascículos. A distribuição variou entre 2 e 16 artigos por fascículo, mas 26 dos 28 fascículos examinados publicaram seis ou mais artigos. $\mathrm{O}$ fascículo com apenas dois artigos traz um índice remissivo entre 1972 e 1993, que ocupa quase todas as suas páginas. Não parece haver ligação entre a quantidade de artigos publicados por fascículo e ano de publicação, pois os fascículos que publicaram quantidade mais elevada de artigos (10 ou mais) estão espalhados ao longo da década (figura 1). 


\section{Autores}

Características da autoria: autoria única e em colaboração

A autoria única predomina sobre autoria em colaboração, confirmando tendência das ciências sociais já verificada em vários estudos (Arenas 2000). Na área específica de ciência da informação, Mueller et alii, (2000) analisaram os anais de quatro encontros da Associação Nacional de Pesquisa e Pós-Graduação em Ciência da Informação (EnAncibs), realizados entre 1994 e 2000, e verificaram a mesma tendência nos trabalhos apresentados nos três últimos encontros:

- EnAncib 1994 - 50\% autoria única e 50\% autoria múltipla;

- EnAncib 1995 - 77,19\% autoria única e 22,81 \% autoria múltipla;

- EnAncib 1997 - 70,15\% autoria única e 29,85 \% autoria múltipla;

- EnAncib 2000 - 75,20\% autoria única e 24,80 \% autoria múltipla.

No caso presente, 194 dos 248 artigos (78,23\%) foram escritos por autor único e 54 artigos $(21,77 \%)$ por mais de um autor, proporção semelhante à encontrada nos três últimos EnAncibs. Os dados ora levantados na Ciência da Informação 1990-1999 revelam que a colaboração entre dois autores foi a forma mais freqüente $(12,90 \%)$ de autoria entre os 54 trabalhos escritos por mais de um autor (figura 2). A soma dos artigos escritos por mais de dois autores chega a apenas $8,47 \%$ do total do universo, com a intensidade de freqüência em ordem inversa ao número de autores.

\section{Produtividade de autor}

Foram registrados 270 autores para os 248 artigos publicados no período estudado. Dentre esses autores, 225 $(83,33 \%)$ - como autores únicos ou em parcerias assinaram apenas um artigo e os restantes 45 (16,66\%) como autores únicos ou em parcerias - assinaram entre dois e cinco artigos. Se, para efeito de cálculo, atribuirmos a cada autor um artigo inteiro tantas vezes quantas seu nome aparece como autor único ou em colaboração (e assim aumentarmos artificialmente o número total de artigos para 347), veremos que os dois autores mais produtivos (com cinco artigos cada um) teriam contribuído com 1,44\% do total desses 347 artigos, enquanto os 225 autores que publicaram apenas um artigo teriam contribuído, cada um, com 0,29\% (figura 3).
FIGURA 2

\section{Autoria única e conjunta}

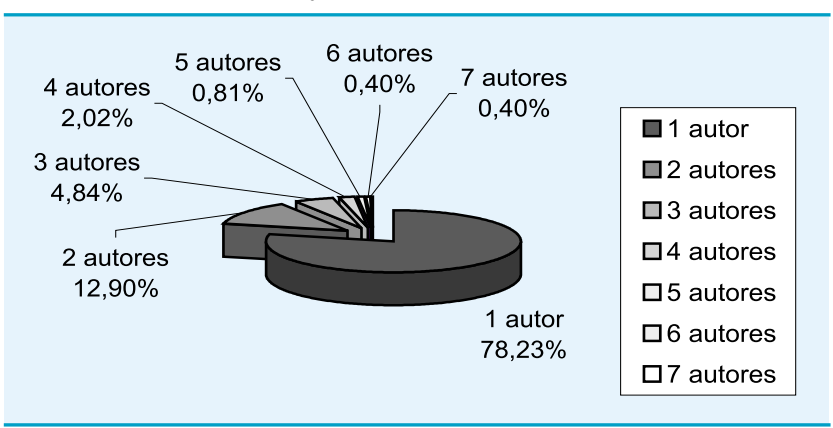

Fonte: Ciência da Informação, 1990-1999

\section{FIGURA 3}

Distribuição da autoria (produtividade de autor)

\begin{tabular}{lllll}
\hline 2 autores & escreveram cada um & 5 & artigos, equivalente à contribuição individual de & $1,44 \%$ do total \\
6 autores & escreveram cada um & 4 & artigos, equivalente à contribuição individual de & $1,15 \%$ do total \\
12 autores & escreveram cada um & 3 & artigos, equivalente à contribuição individual de & $0,86 \%$ do total \\
25 autores & escreveram cada um & 2 & artigos, equivalente à contribuç̧ão individual de & $0,57 \%$ do total \\
225 autores escreveram cada um & 1 & artigo, equivalente à contribuição individual de & $0,29 \%$ do total \\
\hline
\end{tabular}

\section{FIGURA 4}

Contribuição percentual dos autores (autorias únicas e em parcerias)

\begin{tabular}{cccc}
\hline $\begin{array}{c}\text { Número de } \\
\text { Autores } \\
\text { assinando o } \\
\text { artigo }\end{array}$ & $\begin{array}{c}\text { Número de } \\
\text { Artigos } \\
\text { publicados }\end{array}$ & $\begin{array}{c}\% \text { de artigos } \\
\mathrm{n}=248 \text { artigos }\end{array}$ & $\begin{array}{c}\text { Contribuição } \\
\text { individual }\end{array}$ \\
$\begin{array}{c}1 \text { autor } \\
\text { autores }\end{array}$ & 194 & 78,23 & 0,40 \\
\hline autores & 32 & 12,90 & 0,20 \\
4 autores & 5 & 4,84 & 0,13 \\
5 autores & 2 & 2,02 & 0,10 \\
6 autores & 2 & 0,81 & 0,08 \\
7 autores & 1 & 0,40 & 0,07 \\
\hline
\end{tabular}

Fonte: Ciência da Informação, 1990-1999

A contribuição real da cada autor, no entanto, pode ser calculada com maior precisão por meio de ponderação inversa ao número de autores por artigo. Isto é, o autor único teria peso 1 , dois autores escrevendo um artigo teriam peso 0,5 cada um, e assim por diante. Nesse caso, autores que escreveram sozinhos contribuíram, por artigo, o equivalente a $0,40 \%$ do total dos 248 artigos publicados, enquanto a contribuição individual dos autores que escreveram em colaboração variou entre $0,20 \%$ a $0,06 \%$, conforme o número de autores por artigo. Como são apenas 45 os autores que escreveram mais de um artigo (sozinhos ou em colaboração), e considerando que os dois mais produtivos escreveram 5 artigos cada (figura 4), a 
contribuição máxima possível do universo estudado (se o autor tivesse escrito os cinco artigos como autor único) seria de $2 \%$ do total de artigos. Mas, segundo os dados coletados, um dos dois autores mais produtivos escreveu três artigos como autor único e dois em colaboração, enquanto o outro escreveu um artigo como autor único e quatro em colaboração. Logo, o autor mais produtivo do grupo estudado contribuiu com apenas $1,6 \%$ do total. O elevado número de autores e a baixa participação de cada um no universo de artigos publicados na Ciência da Informação na década estudada indicam que os autores estudados ou não escolheram essa revista como veículo freqüente de suas publicações, ou não foram muito produtivos.

\section{Qualificação e origem institucional dos autores mais produtivos}

São os professores dos cursos de pós-graduação na área os autores que mais contribuíram com artigos no período estudado, como pode ser visto na figura 5.

A predominância de professores como autores não pode ser vista com surpresa, pois pesquisar e escrever artigos faz parte da carreira universitária. A presença acentuada dos professores e pesquisadores do curso mantido pelo convênio UFRJ/Ibict indica ligação óbvia entre duas atividades do Ibict, o curso e a revista. Note-se a posição da UFSC, que ainda não tinha curso de pós-graduação no período estudado, e registra um artigo a mais que a Puccamp, cujo curso data da década de 70 , o que talvez se deva a existência da revista Transinformação, editada por este curso. Também chama a atenção a presença de técnicos (não professores ou pesquisadores) e de uma estudante.

A composição da comissão editorial da revista durante o período examinado foi verificada, com o objetivo de identificar a origem institucional dos membros para comparação com a origem institucional dos autores. No entanto, em vários fascículos a origem institucional dos membros não foi citada, frustando assim qualquer comparação. O levantamento com os dados disponíveis e complementado com informações externas apontou a predominância de três instituições na comissão editorial: a UFRJ/lbict, a USP e a UnB. A presença da UFMG também é freqüente, especialmente nos números mais antigos.
FIGURA 5

Qualificação e origem institucional de autores de dois ou mais artigos

\begin{tabular}{|c|c|c|c|}
\hline $\begin{array}{l}\text { Autores : } \\
\text { Qualificação/Instituição }\end{array}$ & $\begin{array}{l}\text { Autores: } \\
\text { Qtdade }\end{array}$ & $\begin{array}{l}\text { Autores : } \\
\text { Qualificação/Instituição }\end{array}$ & $\begin{array}{l}\text { Autores: } \\
\text { Qtdade }\end{array}$ \\
\hline Professores UFRJ/IBICT & 9 & Pesquisador CITEC/IPT-SP & 1 \\
\hline Professores UnB & 7 & Pesquisador IBICT & 1 \\
\hline Professores USP & 5 & Técnico EMBRAPA & 1 \\
\hline Professores UFMG & 4 & Técnico IBICT & 1 \\
\hline Professores UFSC & 3 & Técnico Empresa NATURA & 1 \\
\hline Professores PUCCAMP & 2 & Técnico SENAI/RJ & 1 \\
\hline Pesquisador BIREME & 2 & Técnico USP & 1 \\
\hline Professores UFPr & 1 & Técnico BIREME & 1 \\
\hline Professores Sta. Úrsula & 1 & Bibliotecária & 1 \\
\hline \multirow[t]{2}{*}{ Professores UFF } & 1 & Mestranda UFMG & 1 \\
\hline & & & TOTAL 45 \\
\hline
\end{tabular}

Fonte: Ciência da Informação, 1990-1999

\section{Tendência temática dos artigos}

Para a classificação dos artigos foi utilizada a tabela de temas proposta por Teixeira (1997), com algumas adaptações. A figura A-1 (anexo) mostra as classes maiores com suas subdivisões. Para este trabalho, no entanto, foram utilizadas apenas as nove classes maiores, grafadas em negrito na figura A-1 (anexo); as subdivisões foram utilizadas apenas para orientar a classificação.

A figura 6 mostra a freqüência de temas por classe e também a freqüência de descritores registrados no Library and Information Science Abstract (Lisa) no período estudado. Considerando-se primeiramente apenas os temas encontrados em Ciência da Informação, podemos ver na figura 6 a predominância de dois temas: o Tema 8 - Entrada, tratamento, armazenamento, recuperação e disseminação da informação, com 30,70\% dos artigos, e o Tema 4 - Estudo de usuários, transferência e usos da informação e uso de biblioteca, com 29,87\%. Os demais temas ficaram abaixo de $12 \%$. Não houve artigo sobre o Tema 6 - Prédios de bibliotecas. Se, por um lado, a abrangência dos Temas 8 e 4 poderia explicar a maior incidência de artigos nessas categorias. Por outro, a distribuição apresentada está de acordo com a tradição da biblioteconomia, herdada pela ciência da informação (no Brasil), entre cujas preocupações principais estão o tratamento da informação e a gerência das instituições, como atestaram alguns estudos sobre currículos de formação bibliotecária ${ }^{2}$.

\footnotetext{
${ }^{2}$ Veja, por exemplo, estudos sobre o currículo mínimo e a educação profissional para biblioteconomia realizados nas décadas de 70 e 80 . tais como Lemos (1973), Robredo et alii (1984) e outros.
} 
Na figura 6, estão também dados referentes à freqüência de descritores utilizados pelo periódico de resumo LISA, para artigos publicados no mesmo período, segundo dados obtidos no site da WebSpirs ${ }^{3}$ acessado em 15/1/2001. A diferença em quantidade é muito grande, pois estamos comparando o periódico de resumos Lisa, que indexa publicações do mundo inteiro, com dados de apenas um periódico, mas o uso de percentagens torna os dados comparáveis, pelo menos por grandes assuntos. A comparação mostra não haver coincidência na trajetória das linhas - a Ciência da Informação apresenta distribuição mais equilibrada dos artigos por tema, enquanto LISA apresenta um grande pico para o Tema 2 Teaching of Library Science; Librarian, com mais de $70 \%$ dos descritores, e pouca freqüência relativa para os demais temas. A concentração de descritores no Tema 2 está de acordo com a informação obtida na fonte consultada, mas desperta dúvidas quanto aos critérios utilizados, cuja adequação para a comparação realizada não foi verificada neste estudo.

A distribuição dos temas por ano de publicação está representada na figura 7. Chama a atenção a concentração de artigos sobre determinados temas em alguns anos, como, por exemplo, o Tema 3 - Organização e Gerência de Atividades de Informação, de Bibliotecas e Centros de Pesquisa em 1994 (16 artigos) e em 1999 (10 artigos), o Tema 4 - Estudo de usuários, transferência e usos da informação e uso de biblioteca (zero artigos em 1992 e 18 em 1993), o Tema 5 - Estudo da Literatura e do Documento em 1996 (16 artigos) e 1998 (8 artigos) e o Tema 9 - Outros assuntos em 1995 (11 artigos). O Tema 8 - Entrada, tratamento, armazenamento, recuperação e disseminação da informação - apresenta quantidades altas em vários anos. Examinando-se a tabela no sentido vertical, por ano, podemos verificar concentração de artigos sobre um mesmo tema, sugerindo fascículos temáticos ou quase temáticos, os quais podem ser vistos como indicadores de interesse da época. Da mesma forma, a ausência de artigos sobre o Tema 6, Prédios de bibliotecas, indica falta de interesse que, por sua vez, talvez reflita falta de investimento na construção de prédios, mais comuns em décadas anteriores e que cedeu espaço para bibliotecas digitais e virtuais da década de 90 .
FIGURA 6

Freqüência de artigos por temas - Ciência da Informação e Lisa - 1990-1999

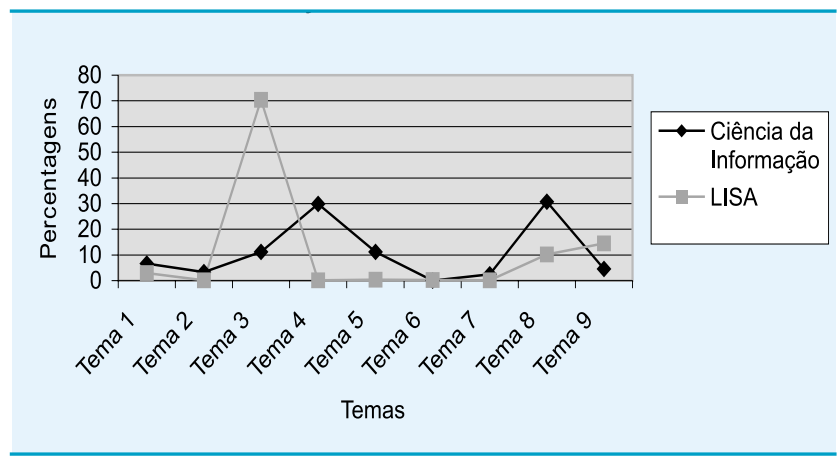

\begin{tabular}{|c|c|c|c|c|c|c|}
\hline $\begin{array}{l}\text { Temas de artigos da Ciência da } \\
\text { Informação, 1990-1999 }\end{array}$ & $\begin{array}{l}\mathrm{N}^{\circ} \mathrm{de} \\
\text { arti- } \\
\text { gos }\end{array}$ & $\%$ & $\begin{array}{l}\text { Codigo } \\
\text { do } \\
\text { tema }\end{array}$ & $\begin{array}{l}\text { Temas indexados no periódico de } \\
\text { resumos LISA 1990-1999 }\end{array}$ & $\begin{array}{l}\mathrm{N}^{\circ} \text { de } \\
\text { arti- } \\
\text { gos }\end{array}$ & $\%$ \\
\hline $\begin{array}{l}\text { Ciência da Informação, Biblioteconomia e } \\
\text { Documentação }\end{array}$ & 16 & 6,63 & 01 & $\begin{array}{l}\text { Information Science, Library } \\
\text { Science and Documentation }\end{array}$ & 306 & 2,90 \\
\hline Ensino, Atividade Profissional e Pesquisa & 8 & 3,35 & 02 & $\begin{array}{l}\text { Teaching if Library Science, } \\
\text { Librarian }\end{array}$ & $\begin{array}{l}14 \\
7442\end{array}$ & $\begin{array}{r}0,13 \\
70,42\end{array}$ \\
\hline $\begin{array}{l}\text { Organização e Gerência de Atividades de } \\
\text { Informação, de Bibliotecas e Centros de } \\
\text { Pesquisa }\end{array}$ & 27 & 11,20 & 03 & $\begin{array}{l}\text { Management of Information } \\
\text { Activities }\end{array}$ & 7 & 0,07 \\
\hline $\begin{array}{l}\text { Estudo de usuário, Transferência e usos de } \\
\text { informação e Uso de Biblioteca }\end{array}$ & 72 & 29,87 & 04 & $\begin{array}{l}\text { User Study. } \\
\text { Information demand }\end{array}$ & $\begin{array}{l}45 \\
23\end{array}$ & $\begin{array}{l}0,43 \\
0,22\end{array}$ \\
\hline Estudo da Literatura e do Documento & 27 & 11,20 & 05 & Literature study & 10 & 0,09 \\
\hline Prédios de Biblioteca & 0 & 0 & 06 & Library Buildings & 1074 & 10,16 \\
\hline Serviços Técnicos & 6 & 2,48 & 07 & $\begin{array}{l}\text { Collection develpmnet and } \\
\text { aquisitions }\end{array}$ & 1531 & 14,49 \\
\hline $\begin{array}{l}\text { Entrada, Tratamento, Armazenamento, } \\
\text { Recuperação e Disseminação da } \\
\text { Informação }\end{array}$ & 74 & 30,70 & 08 & Technical processes & 116 & 1,10 \\
\hline Outros assuntos & 11 & 4,56 & 09 & (não computado) & & \\
\hline Total & 241 & 100 & & & & 100 \\
\hline
\end{tabular}

Fonte: Ciência da Informação, 1990-1999, e LISA, 1990-1999

\section{FIGURA 7}

\section{Distribuição dos temas por ano de publicação}

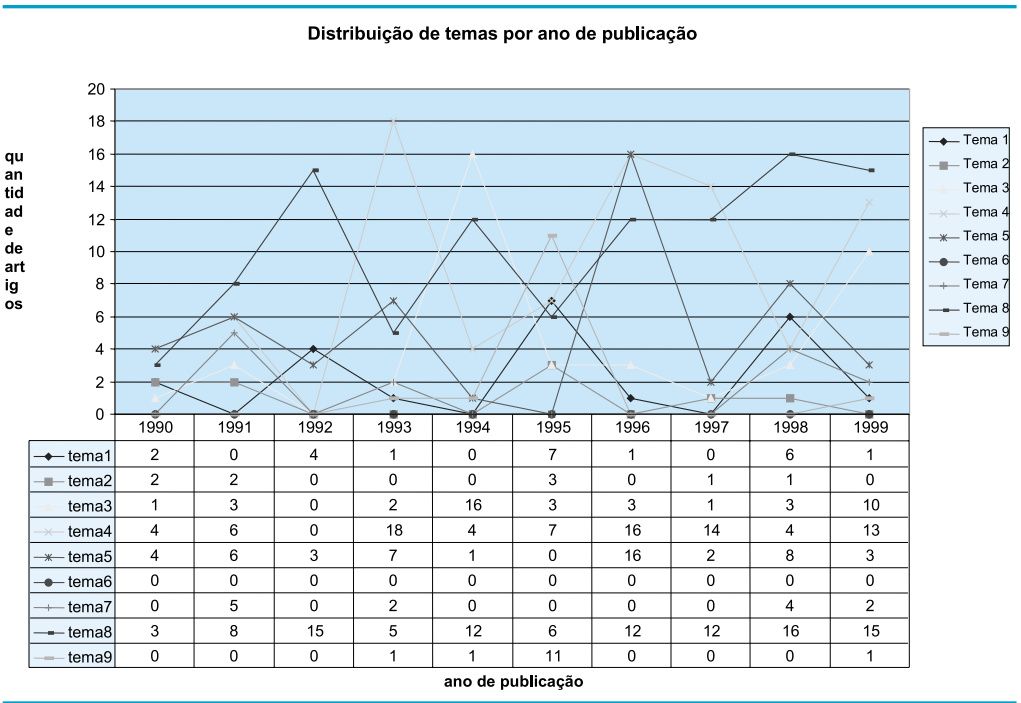

Fonte: Ciência da Informação, 1990-1999

\footnotetext{
${ }^{3}$ http://200.219.29.133:8590/?sp.nextform = history.htm\&sp.usernumber. $\mathrm{p}=351945>$
} 


\section{Grupos de autores por interesse temático}

Os autores foram agrupados por grupos temáticos, isto é, de acordo com as palavras-chave dos artigos que publicaram. Autores de mais de um artigo podem ser classificados em mais de um grupo, o que ocorre quando os artigos que escreveram foram classificados em grupos diferentes. Essa diversidade de interesses ocorreu com freqüência entre os autores que publicaram mais de um artigo. Três foi o maior número de artigos publicados por um mesmo autor sobre um mesmo tema; 16 autores publicaram pelo menos dois artigos no mesmo tema, entre os quais estão nove que publicaram exclusivamente em um mesmo tema. A distribuição está representada na figura 8 .

O Tema 9, Outros assuntos, abrange temas que não se enquadram nos demais grupos e não se repetiram em quantidade suficiente para formar um grupo distinto. O total de 14 trabalhos, no entanto, é elevado para essa categoria sem definição específica e mostra que o leque de assuntos efetivamente tratados é mais amplo do que os temas que foram escolhidos como parâmetros de classificação. Segundo a figura 6, dentre aqueles artigos, 11 foram publicados em 1995, sugerindo um fascículo temático, com tema atípico, ou para os quais não havia previsão na tabela utilizada, indicando necessidade de adequação em estudos posteriores.

Na figura A-2 (anexo 2), os autores foram agrupados conforme os temas de seus artigos. $\mathrm{O}$ fato de que apenas 45 autores contribuíram com mais de um artigo para a revista Ciência da Informação no período de 10 anos faz com que a noção de grupo como "um conjunto de autores que pesquisa os mesmos assuntos" seja tomada com cautela e confirmada com a identificação de pesquisas publicadas por esses autores em outros veículos.

A tabela utilizada para classificação dos artigos mostrouse deficiente para mostrar com a devida ênfase temas mais recentes, ou pelo menos cujas denominações são recentes. Por exemplo, temas como inteligência competitiva, bibliotecas virtuais e outros ficaram imperceptíveis sob cabeçalhos mais amplos e tradicionais, tais como organização e gerência de atividades de informação, de bibliotecas e centros de pesquisa.
FIGURA 8

Distribuição de autores conforme temas de artigos publicados

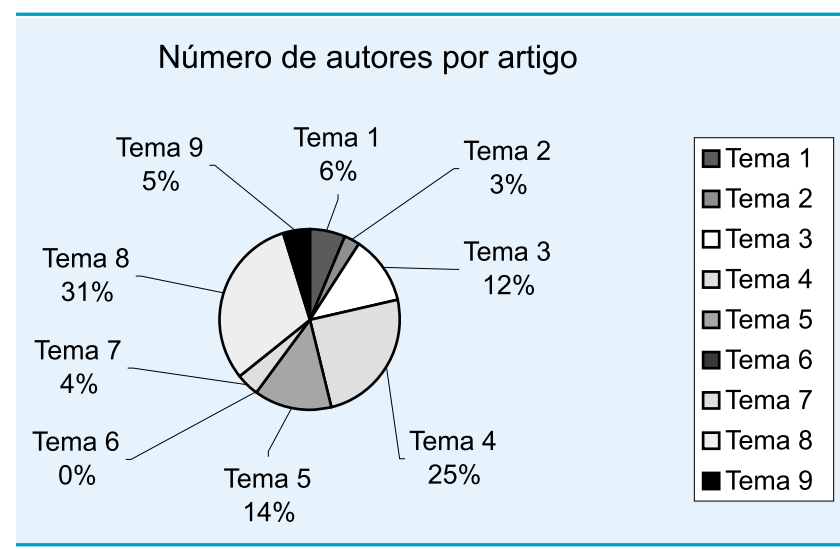

\begin{tabular}{lc}
\hline Grupos temáticos & Número de autores* \\
Tema 1 - Ciência da Informação, Biblioteconomia ... & 19 \\
Tema 2 - Ensino, Atividde Profissional e Pesquisa & 09 \\
Tema 3 - Organização e gerência de atividades de ... & 37 \\
Tema 4 - Estudo de usuário, Transferência e usos de .. & 74 \\
Tema 5 - Estudo da Literatura e do Documento & 42 \\
Tema 6 - Prédios de Biblioteca & 0 \\
Tema 7 - Serviços Técnicos & 13 \\
Tema 8 - Entrada, Tratamento, Armazenamento ... & 94 \\
Tema 9 - Outros assuntos & 14 \\
\hline
\end{tabular}

Fonte: Ciência da Informação, 1990-1999

\section{COMENTÁRIOS FINAIS}

Os resultados obtidos neste levantamento apresentam alguns aspectos interessantes, que serão comentados a seguir:

Embora o universo de 248 artigos publicados na década de 90 não tenha, à primeira vista, distribuído-se uniformemente no período, 20 dentre os 28 fascículos publicaram mais de oito artigos. Esse dado caracteriza a revista como estável, com boa capacidade de atração de artigos. Outras duas revistas da área, fundadas no mesmo período (início da década de 70) e freqüentemente incluídas entre as mais importantes, não apresentaram a mesma estabilidade, tendo sofrido atrasos freqüentes, interrupções ${ }^{4}$ e mudança de nome ${ }^{5}$. A diferença mais óbvia entre as três revistas é a instituição responsável: o órgão responsável pela Ciência da Informação é o Ibict, um instituto de pesquisa vinculado ao Conselho Nacional de Desenvolvimento Científico e Tecnológico (CNPq) e ao Ministério da Ciência e Tecnologia, e as outras duas são publicadas por cursos (departamentos) mantidos por universidades federais.

\footnotetext{
${ }^{4}$ Revista de Biblioteconomia de Brasília.

${ }^{5}$ Revista da Escola de Biblioteconomia da UFMG, hoje Perspectivas em Informação.
} 
Mais de três quartos (78\%) dos indivíduos do universo de 270 autores assinaram seu artigo como autor único. Também é elevado (83\%) o número de autores que publicaram apenas um artigo no periódico Ciência da Informação no período estudado, como autor único ou em colaboração. Os outros $16 \%$ do universo de autores assinaram de dois a cinco artigos (como autor único ou em parceria). A produtividade de autores de uma área do conhecimento não pode ser visualizada com dados de uma só revista científica, por mais prestigiosa e constante que ela seja, mas é interessante notar que 25 desses 45 autores mais produtivos contribuíram com não mais que dois artigos, e os dois autores que mais contribuíram assinaram cinco trabalhos. Poucos autores escreveram mais de uma vez sobre o mesmo tema, de novo sugerindo pesquisa mais abrangente em busca de um quadro mais completo, envolvendo outras fontes.

Foram publicados 54 artigos em parceria de dois a sete autores, uma quantidade baixa que representa aproximadamente $8,5 \%$ do universo de artigos. $O$ grande número de autores que publicaram apenas um artigo não parece ter prejudicado a revista, que conseguiu manter um número elevado de artigos por fascículo durante toda a década. A capacidade de atrair autores tem sido comentada na literatura como um problema comum a muitas revistas nacionais, pois a primeira escolha de muitos pesquisadores são as revistas internacionais, com maior visibilidade no panorama mundial. As agências de fomento, tais como Conselho Nacional de Desenvolvimento Científico e Tecnológico (CNPq) e Fundação Coordenação de Aperfeiçoamento de Pessoal de Nível Superior (Capes) costumam atribuir pontuação mais elevada a artigos publicados nas revistas internacionais quando avaliam cursos de pós-graduação e curriculum vitae de pesquisadores. O fato de (aparentemente) a Ciência da Informação não sofrer com o problema da baixa oferta de artigos mereceria investigação mais aprofundada.

O número de autores que compõem cada grupo temático é bastante diferenciado. Os dois grupos maiores (Tema 8 Entrada, Tratamento, Armazenamento, Recuperação e Disseminação da Informação - e Tema 4 - Estudo de usuário, Transferência e usos de informação e Uso de Biblioteca) têm em comum a preocupação com a organização do conhecimento e de instituições dedicadas à sua custódia, ao uso e ao usuário da informação. A política editorial da Ciência da Informação é abrangente, e não é possível dizer com os dados levantados se essa característica é própria desta revista apenas ou da área como um todo. Para tanto, seria necessário estender o estudo para outras revistas.
A comparação com os descritores do periódico de resumo LISA levantou algumas dúvidas relacionadas à compatibilidade desses dados para comparação e, por conseguinte, à sua validade. De qualquer forma, a própria escolha de descritores fornece um indicador de ênfases. A escolha da Tabela de Teixeira também mostrou deficiências que ainda foram acentuadas, por terem sido adotados apenas os cabeçalhos mais amplos, embora as subdivisões tenham guiado a classificação. Assim, ficaram obscurecidos temas emergentes e presentes na revista nos últimos anos, aos quais foram dedicados fascículos especiais, como é o caso, por exemplo, do v. 26, n. 3, 1997, dedicado ao tema Informação como recurso estratégico para a empresa; v. 27, n. 2, 1998, dedicado ao tema Rumo à publicação eletrônica; v. 28, n. 1, 1999, dedicado ao tema Gerência estratégica do conhecimento; v. 28, n. 2, 1999, dedicado ao tema Políticas e gestão da Informação. Esses temas estão incluídos no levantamento, mas não ficou clara a emergência como assuntos específicos ${ }^{6}$.

O exercício relatado neste artigo mostrou as potencialidades do método utilizado para estudo de literaturas específicas, especialmente artigos científicos. A revista Ciência da Informação tem apresentado papel relevante na literatura da área, e os resultados obtidos são significativos. O levantamento realizado, baseado em dados quantitativos, não pretende explicar as causas dos fenômenos encontrados, mas aponta facetas que poderão ser exploradas em estudos posteriores, por meio de técnicas adequadas. É preciso ter em mente também que, por mais significativa e prestigiosa que a revista Ciência da Informação seja, não é possível generalizar esses resultados para toda a área.

\footnotetext{
${ }^{6}$ Agradecemos a colaboração do avaliador não identificado por levantar este ponto.
} 


\section{Suzana Pinheiro Machado Mueller / Cláudia Maria Pinho de Abreu Pecegueiro}

\section{REFERÊNCIAS BIBLIOGRÁFICAS}

ARENAS et al. Una visión bibliométrica de la investigación en bibliotecologia y ciência de la informacion de la América Latina y Caribe. Revista Española de Documentación Científica, v.23, n.1, 2000.

CHEN, CHING-CHIC. As tecnologias multimídias. In: Informação: tendências para o novo milênio. Brasília : IBICT, 1999. p. 26-47.

JAVERLIN K.; VAKKARI, P. Content analysis of research articles in library and information science. Library and Information Science Research, v. 12 , p. 395-421, 1990.

JAVERLIN K.; VAKKARI, P. The evolution of library and information science 1965-1985: a content analysis of journal articles. Information Processing EO Management, v. 29, n. 1, p.129-144, 1993.

LEMOS, A. A. Briquet de. Estado atual do ensino da biblioteconomia no Brasil e a questão da ciência da informação. Revista de Biblioteconomia de Brasília, Brasília, v.1, n.1, jan./jun. 1973.

MIRANDA, A. Revistas especializadas brasileiras em bibliteconomia e ciência da informação com ênfase da ABDF. Boletim ABDF. Nova série, Brasília, v. 4, n.4, p.30-42, out./dez. 1981.

MIRANDA, D. B. de, PEREIRA, M. de N. F. O periódico científico como veículo de comunicação: uma revisão da literatura. Ciência da Informação, Brasília, v. 25, n. 3, p. 375-382, set./dez. 1996.
MOSTAFA, S. P. Ciência da informação: uma ciência uma revista. Ciência da Informação, Brasília, v. 25, n. 3, p. 305-307, set./dez. 1996

MUELLER et al. O estado da arte da pesquisa em ciência da informação no Brasil: análise dos trabalhos apresentados no IV Enancib, 2000. Disponível em: <http://www.alternex.com.br/ aldoibict/ancib/> Acesso em: $19 \mathrm{dez} .2000$

MUELLER, S. P. M. O círculo vicioso que prende os periódicos nacionais. Datagramazero, v. O, dez. 1999. Disponível em: <http:// www.dgzero.org/dez99/F-I-art.htm >

MUELLER, S. P. M.; CAMPELO, B. S.; DIAS, E. J. W. Disseminação da pesquisa em ciência da informação e biblioteconomia no Brasil. Ciência da Informação, Brasília, v. 25, n. 3, p. 337-352, set./dez. 1996.

ROBREDO, J. et al. Tendências observadas no mercado de trabalho dos bibliotecários e tendências da informação, nas bibliotecas especializadas do Distrito Federal, e qualificações requeridas. Revista de Biblioteconomia de Brasília, Brasília, v. 12, n. 2, jul./dez. 1984

TEIXEIRA, S. K.S. Temática das dissertações defendidas no curso de mestrado em biblioteconomia e documentação da Universidade de Brasília. 1997. 135 f. Dissertação (Mestrado em Ciência da Informação) - Universidade de Brasília, Brasília, [1997?]. 
FIGURA A-1 (ANEXO)

TABELA DAS DISSERTAÇÕES ${ }^{1}$

(distribuição em grandes e pequenos assuntos),

\section{N. ASSUNTOS GERAIS E ESPECÍFICOS}

\section{Ciência da Informação, Biblioteconomia e Documentação (generalidades)}

Estudo comparado (metodologia)/

02 Ensino, Atividade Profissional e Pesquisa

Objetivos educacionais

Metodologia e programa de ensino

Programa de ensino /

Atividade de Pesquisa

Formação Profissional

Profissional da Informação

Auto-imagem profissional

Biografia do Bibliotecário

03 Organização e Gerência de Atividades de Informação, de Bibliotecas e Centros Pesquisa

Consultorias

Padrões de infra-estrutura

Comportamento gerencial

Estilos gerenciais

Motivação

Tomada de decisão

Marketing

Custos

Recursos financeiros

Fluxo da informação

Normalização

Planejamento em sistema de informação

Planejamento de bibliotecas universitárias

Avaliação de sistemas de informação e base de dados

Avaliação de coleções

Automação de bibliotecas

Instituições de informação científica e tecnológica

Arquivos públicos

04 Estudo de Usuário, Transferência e Uso da Informação e Uso da Biblioteca

Caracterização do usuário

Educação e treinamento de usuários

Hábitos de leitura

Transferência, demanda e necessidade de informação

Comunicação, divulgação e editoração científica

Uso da informação e biblioteca

Interempréstimo e serviços de fotocópias (comuntação)

05 Estudos da Literatura e do Documento

Lei de Bradford (substituir por

Estudos de citações

Estudos de citações (metodologia)

Infometria (metodologia)

Avaliação de periódicos

Livros de arte

\footnotetext{
${ }^{1}$ Fonte: Teixeira, 1997
} 


\section{N. ASSUNTOS GERAIS E ESPECÍFICOS}

\section{Prédios de Bibliotecas}

Ambientação

07 Serviços Técnicos

Seleção e aquisição

Desenvolvimento de coleções

Retirada e descarte

Preservação e conservação

Segurança (proteção do acervo)

08 Entrada, Tratamento, Armazenamento, Recuperação e Disseminação da Informação

Controle bibliográfico

Descrição bibliográfica

Formatos bibliográficos

Intercâmbio de registros automatizados

Sistemas cooperativos

Indexação manual e automática (aspectos gerais e teóricos)

Tesauros (aspectos gerais e teóricos)

Elaboração de resumos

Hipertexto

Videotexto

Sistemas especialistas

Redes e sistemas de informação

Recuperação da informação (aspectos gerais e teóricos, buscas)

DSI (avaliação)

Disponibilidade e acessibilidade

Sistema de informação especializados

- Informação agrícola

- Informação ambiental

- Informação científica e tecnológica

- Informação tecnológica

09 Outros Assuntos Correlatos ou adicionais

Ciência da terminologia

Informática

Lingüística

Microinformática

Tradutologia

Traduções (aspectos teóricos e técnicos) 
ANEXO 2 FIGURA A-2 AUTORES POR TEMA

TEMA 1

\section{Autor}

Albagli, Sarita

Braga, Gilda Maria

Christovão, Heloisa Tardin

Costa, Antônio Felipe Corrêa da

Figueiredo, Nice Menezes de

Frota, Maria Guiomar da Cunha

Galvão, Maria Cristane Barbosa

Gomez, Maria Nélida González de

Hagen, Acácia Maria Maduro

Jardim, José Maria

Kondo, Edson Kenji

Lima, Ademir Benedito Alves de

Loureiro, José Mauro Matheus

Maranon, Eduardo Ismael Murguia

Mostafa, Solange Puntel

Mota, Teresa Lenice Nogueira da Gama

Nehmy, Rosa Maria Quadros

Paim, Isís

Pinheiro, Lena Vânia Ribeiro

Saracevic, Tefko

Vieira, Anna da Soledade

Anos
1995
1995
1995
1990
1992
1998
1998
990,1993
1998
1996
1998
1992
1995
1992
1992
1999
1998
1998
1995
1995
1995

Tema
1
1
1
1
1
1
1
1
1
1
1
1
1
1
1
1
1
1
1
1
1

Qtd.

\section{TEMA 2}

\section{Autor}

Barbosa, Ricardo Rodrigues

Cunha, Murilo Bastos da

Eyre, John

Montalli, Kátia Maria Lemos

Mostafa, Solange Puntel

Pacheco, Márcia

Paiva, Denise Werneck de

Souza, Francisco das Chagas de

Souza, Helena de Miranda Rosa e

$\begin{array}{ccc}\text { Anos } & \text { Tema } & \text { Qtd. } \\ 1998 & 2 & \\ 1991 & 2 & \\ 1995 & 2 & \\ 1997 & 2 \\ 1995 & 2 \\ 1995 & 2 \\ 1990 & 2 & \\ 1991 & 2 & \\ 1990 & 2\end{array}$

\section{TEMA 3}

\section{Autor}

Almeida, Iêda Muniz de

Amaral, Sueli Angelica do

Baptista, Dulce Maria

Bordignon, Fernando Raul Alfredo

Borges, Mônica Erichsen Nassif

Carvalho, Kátia de

Carvalho, Natália Guiné de Mello

Cianconi, Regina de Barros

Côrte, Adelaide Ramos e

Cubillo, Julio

Domene, Semíramis Martins Álvares

$\begin{array}{cc}\text { Anos } & \text { Tema } \\ 1999 & 3 \\ 1994 & 3 \\ 1994 & 3 \\ 1999 & 3 \\ 1998 & 3 \\ 1995,1996 & 3 \\ 1998 & 3 \\ 1991 & 3 \\ 1999 & 3 \\ 1997 & 3 \\ 1994 & 3\end{array}$

Qtd.

3

2 
TEMA 3 (continuação)

\section{Autor}

Dórea, José Garrofe

Esmeraldo, Maria Bernadete P.

Fernandes, Pedro Onofre

Figueiredo, Nice Menezes de

Fonseca, Maria Odila

Gregolim, José Angelo Rodrigues

Jardim, José Maria

Lima, Gercina Ângela Borém

Lopes, Ildeu Ordini

Lopes, Luis Carlos

Marchiori, Patricia Zeni

Maura, Mariano A

Miranda, Antônio Lisboa Carvalho de

Nesterov, Anatoly V.

Oliveira, Admar Costa de

Paulino, Ruth Simão

Pellegrini, Ana Emília

Pereira, Maria de Nazaré Freitas

Pinheiro, Ana Virgínia Teixeira da Paz

Pontes, Cecília Carmem Cunha

Quoniam, Luc

Rezende, Yara

Saenger, José Carlos

Santos, Raimundo Nonato Macedo dos

Tolosa, Gabriel Hernan

Valls, Valéria Martins

Vargas, Lilia

\begin{tabular}{|c|c|c|}
\hline Anos & Tema & Qtd. \\
\hline 1994 & 3 & \\
\hline 1999 & 3 & \\
\hline 1991 & 3 & \\
\hline 1995 & 3 & \\
\hline 1999 & 3 & \\
\hline 1994 & 3 & \\
\hline 1998 & 3 & \\
\hline 1999 & 3 & \\
\hline 1999 & 3 & \\
\hline 1994 & 3 & \\
\hline 1994,1994 & 3 & 2 \\
\hline 1993 & 3 & \\
\hline 1993 & 3 & \\
\hline 1991 & 3 & \\
\hline 1994 & 3 & \\
\hline 1994 & 3 & \\
\hline 1999 & 3 & \\
\hline 1995 & 3 & \\
\hline 1990 & 3 & \\
\hline 1994 & 3 & \\
\hline 1994 & 3 & \\
\hline 1994,1994 & 3 & 2 \\
\hline 1999 & 3 & \\
\hline 1994 & 3 & 2 \\
\hline 1999 & 3 & \\
\hline 1996 & 3 & \\
\hline 1994 & 3 & \\
\hline
\end{tabular}

\section{TEMA 4}

\section{Autor}

Aguiar, Afrânio Carvalho

Aguiar, Sonia

Albagli, Sarita

Allevato, Sônia Regina

Alvim, Paulo César Rezende de Carvalho

Amaral, Sueli Angélica do

Araújo, Eliany Alvarenga de

Araujo, Vânia Maria Rodrigues Hermes de

Ataíde, Maria Elza Miranda

Aun, Marta Pinheiro

Barreto, Aldo de Albuquerque

Belluzzo, Regina Célia Baptista

Bemfica, Juliana do Couto

Bernardi, Renato

Bertarello, Maria Ballestrin

Bettiol, Eugênia Maranhão

Borges, Mônica Erichsen Nassif

Cavan, Michael McCarthy

Costa, Icléia Thiesen Magalhães

$\begin{array}{ccc}\text { Anos } & \text { Tema } & \text { Qtd. } \\ 1991 & 4 & \\ 1999 & 4 & \\ 1996 & 4 & \\ 1995 & 4 & \\ 1998 & 4 & \\ 1995 & 4 & 2 \\ 1999 & 4 & \\ 1991,1997 & 4 & \\ 1997 & 4 & \\ 1996,1999 & 4 & \\ 1990,1996,1998,1999 & 4 & \\ 1993 & 4 & \\ 1999 & 4 & \\ 1993 & 4 & \\ 1993 & 4 & \\ 1990 & 4 & \\ 1995,1996,1999 & 4 & \\ 1997 & 4 & \\ 1999 & 4 & \end{array}$




\section{TEMA 4 (continuação)}

\section{Autor}

Cunha, Murilo Bastos da

Cysne, Fatima Portela

Dholakia, Nikhilesh

Dholakia, Ruby Roy

Elhajji, Mohammed

Ferreira, Sueli Mara Soares Pinto

Figueiredo, Nice Menezes de

Fiorese, Venderlei

Freire, Isa Maria

Giacometti, Maria Marta

Gomes, Suely Henrique de A

Gomez, Maria Nélida González de

González, Soray Córdoba

Hubbard, Bruce A

Kielgast, Soeren

King, Donald W.

Klintoe, Kjeld

Lastres, Maria Helena Martins

Launo, Ritva

Leitão, Doradme Moura

Levacov, Marília

Lima, Ademir Benetido Alves de

Lima, Clóvis Ricardo Montenegro de

Lopes, Luis Carlos

Macedo, Neusa Dias de

Marchiori, Patricia Zeni

Marteleto, Regina Maria

Maury, Patrick

Mendes, Teresa Cristina M.

Mendonça, Lícia Maria Enout

Miranda, Roberto Campos da Rocha

Montalli, Kátia Maria Lemos

Moran, José Manuel

Moura, Luciano Raizer

Mueller, Suzana Pinheiro Machado

Mundorf, Norbert

Ottoni, Heloisa Maria

Paternostro, Luiz Carlos Brito

Pinto, Virgínia Bentes

Pontual, Miraci de Arruda Camara

Ramos, Mauro Gonçalves

Ramos, Paulo Baltazar

Robredo, Jaime

Rocha, Eliana da Conceição

Santos Júnior, José Neiva

Schukste, Marília R. C.

Senra, Nelson de Castro

Silva, Gabriela Lopes da

\section{Anos}

1993

1996

1997

1997

1999

1996

1990

1993

$1991,1995,1996,1997$

1990

1993

1995

1998

1997

1997

1998

1991

1995,1996,1999

1993

1993

1997

1992

1993

1993

1993

1997

1995

1993

1997

1992

1999

1991

1994

1996

1994

1997

1996,1996

1996

1993

1994

1994

1996

1993

1993

1996

1993

1997,1999

1997

\section{Tema}

Qtd.

4

4

4

4

4

4

4

4

4

4

3

2

2 


\section{TEMA 4 (continuação)}

\section{Autor}

Silva, Inez Leite Gonçalves da

Souza, Francisco das Chagas de

Souza, Terezinha de Fátima Carvalho de

Tasca, Cláudia Cecília

Tenopir, Carol

Urdaneta, Iraset Páez

Vieira, Anna da Soledade

Wanderley, Ana Valéria Medeiros

$\begin{array}{ccc}\text { Anos } & \text { Tema } & \text { Qtd. } \\ 1996 & 4 & \\ 1991 & 4 & \\ 1996 & 4 & \\ 1993 & 4 & \\ 1998 & 4 & \\ 1992 & 4 & \\ 1993,1999 & 4 & \\ 1999 & 4 & \end{array}$

\section{TEMA 5}

\section{Autor}

Alvarenga, Lídia

Andrade, Maria Eugênia Albino

Aragão, Esmeralda Maria de

Barbeitos, Marcos

Barros, Edgard de Vasconcelos

Bressan, Matheus

Campello, Bernadete Santos

Castro, Regina Célia F.

Coelho, Ricardo M. Pinto

Coutinho, Eliana

Couzinet, Viviane

Currás, Emilia

Dias, Eduardo José Wense

Duarte, Francisco A de Moura

Ferreira, Maria Cecília Gonzaga

Krieger, Eduardo Moacyr

Krzyzanowski, Rosaly Favero

Lima, Maria de Fátima Bezerra Ferreira

Macias-Chapula, Cesar A

Medeiros, Nilcéia Lage de

Mello, Paula Maria Abrantes Cotta de

Menezes, Estera Muszkat

Miranda, Antônio Lisboa Carvalho de

Mostafa, Solange Puntel

Mueller, Suzana Pinheiro Machado

Muniz, José Norberto

Negrão, May Brooking

Noronha, Daisy Pires

Oberhofer, Cecília Malizia A

Oliveira, José Almir Martins

Oliveira, Margarida Pinto

Oliveira, Maria José de

Pereira, Maria de Nazaré Freitas

Poblacion, Dinah Aguiar

Rodrigues, Maria da Paz Lins

Rousseau, Ronald

Silva, Eduardo Orozco

\section{ANOS}

1998

1993

1992

1997

1990

1990

1993,1996

1996,1996

1997

1991

1999

1996

1996

1991

1996,1998

1991

1991,1998

1993

1998

1993

1996

1999

1996

1993

1991,1996,1996

1990

1996

1996,1998

1991

1990

1992

1994

1996

1996

1993

1998

1999
TEMA

5

5

5

5

5

5

5

5

5

5

5

5

5

5

5

5

5

5

5

5

5

5

5

5

5

5

5

5

5

5

5

5

5

5

5

5

5 
TEMA 5 (continuação)

\section{Autor}

Spinak, Ernesto

Stumpf, Ida Regina Chitto

Trzesniak, Piotr

Vidili, Ana Lúcia

Zaher, Celia Ribeiro

$\begin{array}{ccc}\text { Anos } & \text { Tema } & \text { Qtd. } \\ 1998 & 5 & \\ 1996 & 5 & \\ 1998 & 5 & \\ 1996 & 5 & \\ 1996 & 5 & \end{array}$

TEMA 7

\section{Autor}

Borges, Mônica Erichsen Nassif

$\begin{array}{ccc}\text { Anos } & \text { Tema } & \text { Qtd. } \\ 1999 & 7 & \\ 1998 & 7 & \\ 1998 & 7 & \\ 1991 & 7 & \\ 1998 & 7 & \\ 1993 & 7 & \\ 1991 & 7 & \\ 1991 & 7 & \\ 1991 & 7 & \\ 1991 & 7 & \\ 1998 & 7 & \\ 1999 & 7 & \\ 1993 & 7 & \end{array}$

Caldeira, Paulo da Terra

Campello, Bernadete Santos

Klaes, Rejane Raffo

Mangue, Manual Valente

Miranda, Antônio Lisboa Carvalho de

Prysthon, Cecília F.

Rocha, Enivaldo C. da

Rocha, Jacira Guiro C. da

Schmidt, Susana

Silva, Aparecida Imaculada Bedeti da

Souza, Terezinha de Fátima Carvalho de

Vergueiro, Waldomiro de Castro Santos

\section{TEMA 8}

\section{Autor}

Albagli, Sarita

Albuquerque, Maria Elizabete B. C.

Antônio, Irati

Araujo, Vânia Maria Rodrigues Hermes

Battaglia, Maria da Glória Botelho

Boccato, Vera Regina Casari

Brito, Marcílio de

Campos, Maria Luiza Almeida

Caribé, Rita de Cássia do Vale

Carlisie, Judith

Carmo, Vadson Bastos do

Carvalho, Márcia Medeiros de

Cianconi, Regina de Barros

Cuenca, Angela Maria Belloni

\section{Anos}

1998

1998

1998

1995

1999

1996

1992

1996

1992

1999

1999

1996

1990,1994

1999

Cunha, Murilo Bastos da

Cunningham, Scott

$1994,1998,1999$

1999

1992

1999

1994

1994

1998

1992

1994

1994

\section{Tema}

8

8

8

8

8

8

8

8

8

8

8

8

8

2

8

8

8

8

8

8

8

8

8

8

8 


\section{TEMA 8 (continuação)}

\section{Autor}

Freitas, Henrique M. R. de

Furnival, Adriane Chloë

Furtado, João Salvador

Galvão, Alexander Patez

Garcia, Joana Coeli Ribeiro

Gigante, Maristela Cid

Gontow, Rejane

González, José Antônio Moreiro

Guedes, Vânia Lisbôa da Silveira

Jannuzzi, Celeste Aída Sirotheau Corrêa

Kobashi, Nair Yumiko

Kuramoto, Hélio

Lara, Marilda Lopes Ginez de

Lastres, Helena Maria Martins

Lautré, Evelyne

Lima, Vânia Mara Alves

Lopes, Roseane Regina Velho

Lucas, Clarinda Rodrigues

Maloff, Joel

Mamfrim, Flávia Pereira Braga

Mariotto, Fábio Luiz

Martins Filho, Plínio

Melo, Denise Gomes Pereira de

Melo, Maria de Lourdes de Arruda

Mendes, Raquel Dias

Milidiú, Ruy Luiz

Montalli, Kátia Maria Lemos

Mueller, Charles Curt

Naves, Madalena Martins Lopes

Nayak, Anustup

Neves, Dulce Amélia de Brito

Oliveira, Marlene de

Pacheco, Fernando Flávio

Packer, Abel Laerte

Pasquarelli, Maria Luiza Rigo

Passarelli, Brasiliana

Petrini, Maira

Pilnik, Marcia

Pinheiro, Lena Vânia Ribeiro

Pinheiro, Marisa Gurjão

Pohlmann Filho, Omer

Pontes, Cecília Carmem Cunha

Pontes, Cecília Carmem Cunha

Porter, Alan

Pozzebon, Marlei

Prati, Suely Cafazzi

Raabe, André

Rezende, Yara

Ribeiro, Gustavo Lins

\section{Anos}

1997

1995,1996

1991

1999

1998

1996

1997

1998

1994

1999

1992

1996

1992,1993

1995

1992

1996

1991

1997

1997

1991

1992

1998

1998

1998

1997

1991

1994,1999

1992

1998

1999

1998

1996

1991

1993,1998

1995

1993

1997

1996

1994

1991

1998

1999

1999

1999

1997

1996

1998

1990

1992

\section{Tema}

8

8

8

8

8

8

8

8

8

8

8

8

8

8

8

8

8

8

8

8

8

8

8

8

8

8

8

8

8

8

8

8

8

8

8

8

8

8

8

8

8

8

8

8

8

8

8

8

8
Qtd.

2

2

2

2 


\section{TEMA 8 (continuação)}

Autor

Robredo, Jaime

Rosetto, Marcia

Santos, Vanda Ferreira dos

Schiel, Ulrich

Silva, Wagner Teixeira da

Skolimovski, Edith Busolo

Tálamo, Maria de Fátima G.M.

Tarapanoff, Kira

Targino, Maria das Graças

Teixeira, Cenidalva Miranda de Sousa

Thiollent, Michel

Valle, Benjamin de Medeiros

Vargas, José Israel

Viana, Cassandra Lúcia de Maya

Vianna, Rogério Antônio Sampaio Parente

Vieira, Anna da Soledade

Vilan Filho, Jayme Leiro

Viruez, Guilma Vidal

Yamashita, Marina Mayumi

Zaher, Celia Ribeiro

Zhu, Donghua
Anos

1991,1998

1997,1997

1997

1997

1991

1992

11992,1995

1993

1995

1997

1992

1996

1994

1990

1992

1992

1994

1994

1996

1993

1999

\section{Tema}

8

8

8

8

8

8

8

8

8

8

8

8

8

8

8

8

8

8

8

8

8

\section{TEMA 9}

\section{Autor}

Alves, Ieda Maria

Barbosa, Maria Aparecida

Bevillacqua, Cleci Regina

Boulanger, Jean-Claude

Bresciani Filho, Ettore

Cabré, M. Teresa

Correia, Margarita

Faulstich, Enilde

Finatto, Maria José Bocorny

Frota, Maurício Nogueira

Krieger, Maria da Graça

Lins, Bernardo F. E.

Maciel, Anna Maria Becker

Octaviano, Vera Lúcia de Campos

\section{Anos}

1995

1995

1995

1995

1999

1995

1995

1995

1995

1994

1995

1993

1995

1995
Tema

9

9

9

9

9

9

9

9

9

9

9

9

9 